\title{
Development of a Field Cycling NMR System for PQR Detection in Biopolymers*
}

\author{
Dmitri Ivanov and Alfred Redfield \\ Biochemistry Department, Brandeis University, Waltham MA 02254, USA
}

Z. Naturforsch. 53a, 269-272 (1998); received October 31, 1997

\begin{abstract}
Our goal is to extend the sensitivity of field cycling pure quadrupole resonance (PQR) methods to be of use in biological systems. The nuclei of interest are ${ }^{25} \mathrm{Mg},{ }^{67} \mathrm{Zn},{ }^{43} \mathrm{Ca},{ }^{11} \mathrm{~B}$ and ${ }^{17} \mathrm{O}$. The experiment is based on a field cycling double resonance technique, in which the quadrupole resonance of a rare nucleus is found through its effect on the magnetic order of the abundant nucleus to which the rare nucleus is coupled through dipole-dipole interaction. A field-cycling NMR spectrometer has been developed, based on our existing $500 \mathrm{MHz}$ high resolution spectrometer. The sample can be shuttled pneumatically from the high field of a commercial $500 \mathrm{MHz}$ magnet to the magnet's top, where the residual field and its gradient is canceled out by a pair of Helmholtz coils. Low field homogeneity is within 0.5 gauss. The ${ }^{1} \mathrm{H}$ signal is observed at high field as a free induction decay (FID) after a $90^{\circ}$ pulse. At low field the sample can be irradiated by a digitally tuned RF coil in the $300 \mathrm{kHz}-7 \mathrm{MHz}$ range. The sample has to be maintained at low temperature $(\sim 30 \mathrm{~K})$ to avoid relaxation via thermal motion of methyl groups in biomolecules. For this purpose field cycling equipment is placed in a variable temperature dewar $(4-300 \mathrm{~K})$. We plan to use solutions of biomolecules in standard cryoprotective buffer, containing $\sim 30 \%$ glycerol. Preliminary results on the quadrupole resonance of natural abundance ${ }^{17} \mathrm{O}$ in the cryoprotective buffer and of natural abundance ${ }^{11} \mathrm{~B}$ in a protease inhibitor at $50 \mathrm{mM}$ are presented.
\end{abstract}

\section{Introduction}

Since the original experiments of Pound and Ramsey, field cycling NMR has been applied to a variety of systems. See [1,2] for a review and [3] for recent references. The field cycling double resonance method developed by one of us and independently by Slusher and Hahn in the 1960's allows high sensitivity detection of quadrupole resonance. The double resonance nature of the experiment overcomes inherently low sensitivity associated with direct detection of quadrupole signals. The resonance of interest is observed through its effect on the strong signal of an abundant reporter nucleus, in our case protons, after field cycling. The fact that the quadrupole transitions are observed at zero field is also an advantage, because one can use powdered samples without having quadrupole lines broadened due to random relative orientation of the external magnetic field and the local electric field gradient (EFG) tensor. The most sensitive version of the experiment, so-called spin mixing in the laboratory frame, is applicable only to half-integer

* Presented at the XIVth International Symposium on Nuclear Quadrupole Interactions, Pisa, Italy, July 20-25, 1997.

Reprint requests to Prof. Alfred Redfield, Fax: 1-7 81-7362349. spin nuclei, and although half-integer spin nuclei are much more numerous than integer spin ones, that leaves out such biologically interesting nuclei as ${ }^{14} \mathrm{~N}$ and the deuteron. The sensitivity of the method is very high, for example Hsieh, Koo, and Hahn [4] were able to detect ${ }^{17} \mathrm{O}$ signal in cloroquinones and several other organic compounds at natural abundance $(0.037 \%)$ with high signal to noise ratio. Unfortunately the method has never been applied to biological systems despite its sensitivity. Our primary goal is to detect quadrupole signals of such half-integer spin nuclei as ${ }^{67} \mathrm{Zn},{ }^{25} \mathrm{Mg},{ }^{43} \mathrm{Ca},{ }^{11} \mathrm{~B}$ and ${ }^{17} \mathrm{O}$ in proteins or in small molecules bound to proteins, and to evaluate their utility. These isotopes can be detected at natural abundance or enriched at a reasonable cost. The metal ions, which function as an integral part in a variety of biomolecules, have no characteristic features that can be readily studied by currently available spectroscopic methods, other than line-broadening in the solution state, or a few specialized methods. Up to now the usual approach has been to replace these metals with similar ions that can be studied by NMR, EPR or optical spectroscopy. In this respect the quadrupole signals of these nuclei, which are informative of the immediate electric environment of the nucleus, can provide valuable clues about the principles or metal coordination in macromolecules and their 
involvement in biocatalysis. NQR of ${ }^{11} \mathrm{~B}$ and ${ }^{17} \mathrm{O}$ in enzyme bound substrates and inhibitors may help to elucidate structural changes induced by the active site of the enzyme to facilitate chemistry.

\section{Experiment and Apparatus}

Nuclear double resonance experiments are especially useful when the nucleus of interest is present in the sample at a very low concentration, or when a low frequency transition is to be detected. A diagram of a field cycling double resonance experiment is shown in Figure 1. At the beginning or each field cycle of the experiment the sample is polarized in high field $H_{\text {high }}$ for the time $t_{\mathrm{H}}$ on the order of spin-lattice relaxation time $T_{1}$ of protons. After that the sample is moved to zero field for the time $t_{\mathrm{L}}$ (more than $1 \mathrm{sec}$ ). Once in zero field, the sample is irradiated by an RF search field, the frequency of which is incremented every experimental cycle to cover the range of the expected quadrupole resonance frequencies. Finally, the sample is returned back to high field and the residual magnetization of protons is measured by observing an FID after a $90^{\circ}$ pulse.

If the sample is demagnetized adiabatically, that is when $\mathrm{d} H / \mathrm{d} t \ll \gamma\left(H_{\mathrm{L}}^{2}+H^{2}\right)$, where $H_{\mathrm{L}}$ is the mean local field experienced by protons due to dipole-dipole interaction, the initial magnetic order is stored, in zero field, along local dipolar fields. Almost full initial magnetization can be restored after the sample is returned back to high field, given that $t_{\mathrm{L}}$ is less than the zero field spin-lattice relaxation time $T_{1 \mathrm{~d}}$ [5]. In general $T_{1 \mathrm{~d}}$ is shorter than $T_{1}$ at high field, but it can be fairly long in the absence of thermal motion. Samples of macro-

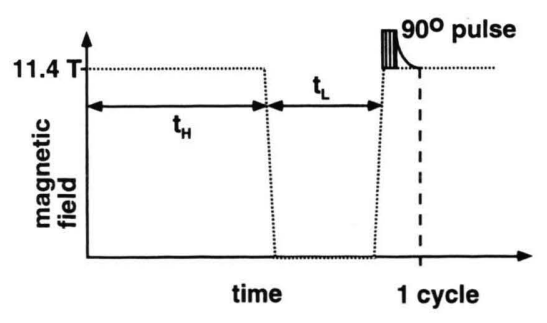

search RF field

Fig. 1. Field-cycling double resonance experiment for PQR detection. The search RF field is usually modulated as described in text. molecules have to be frozen to $<50 \mathrm{~K}$ to stop thermal rotation of methyl groups, which is still present at liquid nitrogen temperature, to make $T_{1 \mathrm{~d}}$ long.

Referring to protons as spins A and to quadrupolar nuclei as spins $Q$, we can write for the proton spin temperature after adiabatic demagnetization

$$
T_{\mathrm{A}}(0) \approx\left(H_{\mathbf{L}} / H_{\text {high }}\right) T_{\mathbf{L}},
$$

where $T_{\mathrm{L}}$ is lattice temperature. When the search $\mathrm{RF}$ field is not at the frequency of quadrupole transition, the two systems A and Q are not in thermal contact and the proton temperature is only affected by zero field spin-lattice relaxation:

$$
T_{\mathrm{A}}(t)=T_{\mathrm{A}}(0) \exp \left\{t / T_{1 \mathrm{~d}}\right\} .
$$

When the search RF field is on resonance $v_{\mathrm{RF}}=v_{\mathrm{Q}}$, and the approximate Hartmann-Hahn condition is observed: $\gamma_{\mathrm{Q}} H_{\mathrm{RF}}=\gamma_{\mathrm{A}} H_{\mathrm{L}}$, the two systems are thermally coupled and some of proton spin order is transferred to the quadrupolar system, as shown by Landesman and Goldman [6], and for proton spin temperature:

$$
T_{\mathrm{A}}(t)=T_{\mathrm{A}}(0) \exp \left\{\left(t / T_{1 \mathrm{~d}}+R_{\mathrm{AQ}}\right)\right\},
$$

where $R_{\mathrm{AQ}}$ is the rate at which order is being exchanged between spins A and Q. Magnetization can flow from spins $A$ to spins $Q$ until they come to a "common spin temperature". To achieve high sensitivity detection of the quadrupole resonance one can phase reverse the RF search field every time the equilibrium is reached [7], heating up the quadrupolar system and destroying another fraction of proton initial magnetization; alternatively one can use frequency modulation of the search RF [8] or use a double frequency irradiation [9]. That will result in a substantial destruction of proton magnetization, and one will see a trough in the plot of proton FID intensity vs. the frequency of RF search field, corresponding to the frequency of quadrupole resonance.

Theoretical calculations for $R_{\mathrm{AQ}}$ predict very high sensitivity for the method, but the actual sensitivity can be limited by a number of factors: quadrupole resonance broadening due to sample heterogeneity, experimental limitations on the strength of the search $\mathrm{RF}$ field, irreproducible positioning of the sample in the probe after physically moving it to zero field. The sensitivity is hard to estimate because all previous work of this kind was done at higher concentrations, lower magnetic fields and higher temperatures. The method works better when the quadrupole frequency 
is higher than $1 \mathrm{MHz}$, because of direct excitation of the protons at low frequency. We are currently trying different methods of RF search field modulation and hope to be able to increase the sensitivity of the method at lower frequencies.

A technical problem was to find relaxing agents and sample temperatures for which the spin-lattice relaxation times in high and low field will have suitable values. The zero field relaxation time of protons $T_{1 \mathrm{~d}}$ has to be a second or two in order to have the time required for sensitive detection of the quadrupole signal. At the same time the relaxation time at high field should be short enough so that the full frequency scan can be completed in a reasonable amount of time. To avoid fast thermal relaxation at zero field we keep the sample temperature at $\sim 30 \mathrm{~K}$. We are using $>30 \%$ glycerol in water solution as cryoprotectant to avoid crystallization of water in biological samples. The samples also have to be purged with argon gas before freezing, because otherwise relaxation times are short due to paramagnetic molecular oxygen dissolved in the sample. Following Hahn, we add about $0.1 \mathrm{mM}$ $\mathrm{Ni}^{2+}$ ion to shorten $T_{1}$ at high field. The resulting $T_{1 \mathrm{~d}}$ is about $1 \mathrm{sec}$, and the high field $T_{1}$ is $5-10 \mathrm{sec}$.

Our field-cycling apparatus is controlled by the existing console of our home-made high resolution $500 \mathrm{MHz}$ spectrometer. The timing and computer analysis systems of the spectrometer are versatile enough to run the quadrupole resonance experiments without a major modification. A commercial variable temperature dewar fits into the $43 \mathrm{~mm}$ bore of the $500 \mathrm{MHz}$ magnet. The inhomogeneity of the unshimmed field of the magnet is less than $1 \mathrm{kHz}$, which is more than sufficient for the application. The sample transfer system is placed in the dewar and mates the upper flange of the dewar. A $500 \mathrm{MHz}$ probe is located on the high field end of the transfer system and is used to observe the proton FID after a $90^{\circ}$ pulse. Two sapphire trimmer capacitors are mounted adjacent to the coil and have long shafts attached to them, so that the probe can be tuned and matched after the equipment is cooled down to the operating temperature. The sample is shuttled pneumatically, using helium gas, a distance of about $1 \mathrm{~m}$ to the magnet's top, where the residual field and its gradient are canceled by a pair of Helmholtz coils. The transit time of the sample is $\sim 0.3 \mathrm{sec}$ each way. The homogeneity of the zero field is 0.5 gauss. By adjusting the current through the coils one can vary the low field in the range of several hundred gauss for future use. The sample is

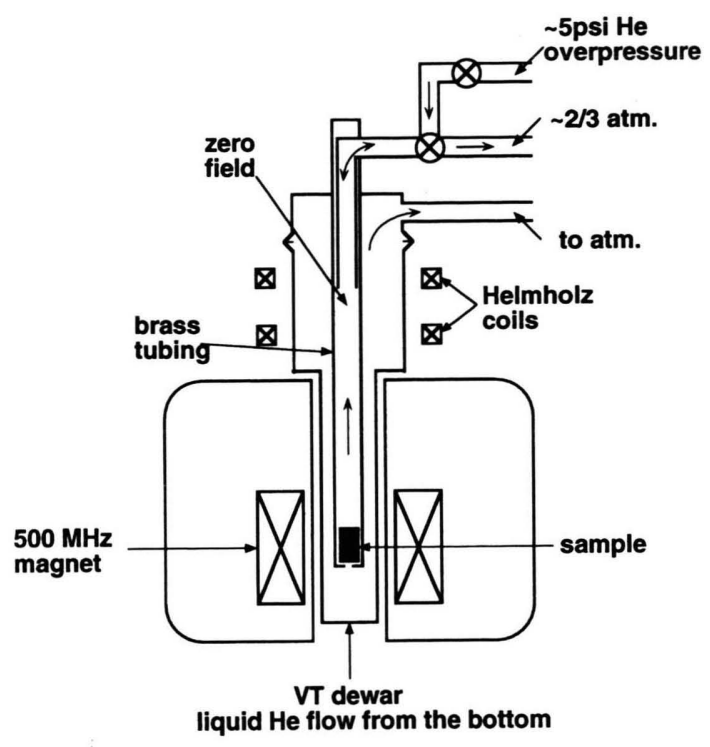

Fig. 2. Experiment setup. The figure is simplified and not to scale.

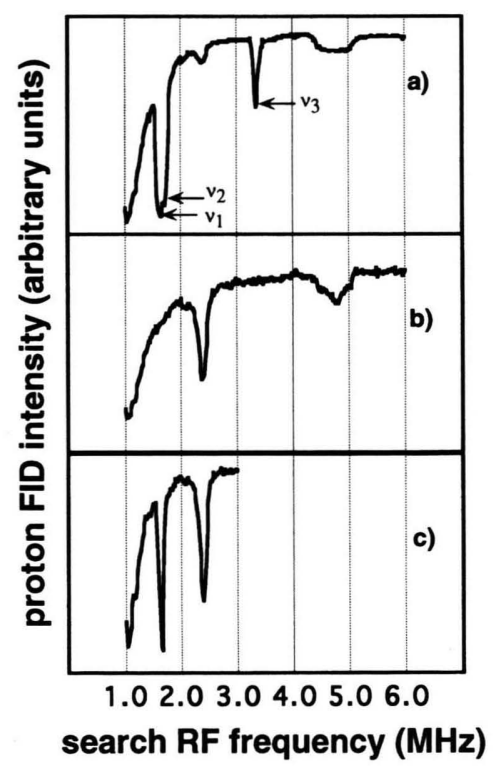

Fig. 3. Spectra of natural abundance ${ }^{17} \mathrm{O}$ and ${ }^{11} \mathrm{~B}$. All experiments were run at $30 \mathrm{~K}$. The search RF was sin-wave modulated using a double-balanced mixer. Modulation frequency was $80 \mathrm{kHz}$. Time at zero field $t_{\mathrm{L}}=1.5 \mathrm{sec}$. (a) Spectrum of $30 \%$ glycerol solution in water. $v_{1}, v_{2}$ and $v_{3}$ correspond to ${ }^{17} \mathrm{O} 1 / 2 \leftrightarrow 3 / 2,3 / 2 \leftrightarrow 5 / 2$ and $1 / 2 \leftrightarrow 5 / 2$ pure quadrupole transitions in water, respectively. (b) ${ }^{17} \mathrm{O}$ spectrum of pure ( $>99.7 \%$ ) glycerol. (c) ${ }^{11} \mathrm{~B}$ spectrum of a $50 \mathrm{mM}$ solution of 1-butyl boronic acid in pure glycerol. Spectra are not on the same scale. 
placed in a Teflon sample holder having a sample space of $4 \mathrm{~mm}$ ID and $1.5 \mathrm{~cm}$ length.

The tuning and the matching capacitors of the zero field coil are digitally controlled, so that the coil can be tuned and matched automatically in the $300 \mathrm{kHz}$ to $7 \mathrm{MHz}$ range.

\section{Preliminary Results}

Figure 3 shows preliminary spectra of natural abundance ${ }^{17} \mathrm{O}$ in water and glycerol $(<20 \mathrm{mM})$ and ${ }^{11} \mathrm{~B}$ in a protease inhibitor at $50 \mathrm{mM}$ concentration. In water the electric field gradient on the ${ }^{17} \mathrm{O}$ nucleus does not have axial symmetry and one can observe $3 / 2 \leftrightarrow 1 / 2,5 / 2 \leftrightarrow 3 / 2$ transitions as well as $5 / 2 \leftrightarrow 1 / 2$ transition, which is forbidden for axially symmetric

[1] F. Noak, Progr. NMR Spectrosc. 18, 171 (1986).

[2] D. T. Edmonds, Physics Reports 29, 234 (1977).

[3] A. G. Redfield, NMR as a Structural Tool for Macromolecules: Current Status and Future Directions, B. D. N. Rao \& M. D. Kemple, eds., Plenum, New York 1995, P. 382 .

[4] Y. N. Hsieh, J. C. Koo, and E. L. Hahn, Chem. Phys. Lett. 13, 563 (1972).
EFG (Figure $3 \mathrm{a}$ ). The frequencies of transitions are very close to the previously reported frequencies of ${ }^{17} \mathrm{O}$ in enriched ice [10]. We believe that even in $30 \%$ glycerol solution water forms local ice-like structures. Water lines get broader if higher concentrations of glycerol are used (data not shown).

1-Butyl boronic acid, the spectrum of which is shown in Fig. 3c, is a member of a class of protease inhibitors. Boronic acid inhibitors bind to a variety of trypsin-like proteases including members of blood clotting system thrombin, plasma kallikrein and plasmin. Some compounds of this class were shown to act as anticoagulants and inflammation inhibitors in rats. The mechanism of inhibition and the details of the enzyme-inhibitor complex are not yet completely clear. We have not tried a metalloprotein or enzyme with a boronic acid inhibitor, but expect to do so soon.

[5] R. V. Pound, Phys. Rev. 81, 156 (1951).

[6] M. Goldman and A. Landesman, Phys. Rev. 132, 610 (1963).

[7] S. Hartmann and E. L. Hahn, Phys. Rev. 128, 2024 (1962)

[8] R. E. Slusher and E. L. Hahn, Phys. Rev. 166, 332 (1968).

[9] A. G. Redfield, Phys. Rev. 130, 589 (1963).

[10] D. T. Edmonds and A. Zussman, Phys. Letters 41 A, 167 (1972). 